American Journal of Applied Sciences 9 (5): 772-778, 2012

ISSN 1546-9239

(C) 2012 Science Publications

\title{
Sequential Multiple Response Optimization for Manufacturing Flexible Printed Circuit
}

\author{
Pichpimon Kanchanasuttisang and Pongchanun Luangpaiboon \\ Department of Industrial Engineering, Faculty of Engineering, \\ Industrial Statistics and Operational Research Unit, \\ Thammasat University, Pathumthani, 12120, Thailand
}

\begin{abstract}
Problem statement: Flexible Printed Circuit or FPC, one of automotive electronic parts, has been developed for lighting automotive vehicles by assembling with the LED. The quality performances or responses of lighting vehicles are relied on the circuit width of an FPC and the etched rate of acid solution. According to the current operating condition of an FPC company, the capability of the manufacturing process is under the company requirement. The standard deviation of FPC circuit widths is at higher levels and the mean is also worse than specifications. Approach: In this process improvement there was four sequential steps based on the designed experiments, steepest descent and interchangeable linear constrained response surface optimization or IC-LCRSOM. An investigation aims to determine the preferable levels of significant process variables affecting multiple responses. Results: The new settings from the IC-LCRSOM improved all performance measures in terms of both the mean and the standard deviation on all process patterns. Conclusion: From this sequential optimization the developed mathematical model has tested for adequacy using analysis of variance and other adequacy measures. In the actual investigation, the new operating conditions lead to higher levels of the etched rate and process capability including lower levels of the standard deviation of the circuit widths and etched rate when compared.
\end{abstract}

Key words: Flexible printed circuit, circuit width, etched rate, multiple regression, linear constrained response surface optimization

\section{INTRODUCTION}

Flexible Printed Circuits (FPC) play a major role in all electronics industries. Nowadays, the market requires increasingly complicated and sophisticated electronic parts in terms of specifications (Tong et al., 2004). Therefore, quality excellence is very critical in the manufacture of flexible printed circuits. A collection of designed experiments and mathematical programming techniques have been applied for quality improvement in automotive electronic parts. Here, a case study on quality improvement of FPC through the systematic approach is presented. FPC is usually applied to mechanically support and electrically connect electronic components. This study particularly interests to improve the quality of the FPC which assembly with the LED for the lighting automotive vehicles.

The emission light and optical properties are mainly relied on the relationship of the etched rate of acid solution and circuit width, one of the key failure and break down to LED of lighting vehicles. The principles of the upward acid spray and the use of additives to reduce the etching ability are necessary for successful implementations (Coombs, 2007). So to validate on processing and to sustain finished goods with the permanent prevention, the precisely etched condition should be optimized. On characteristics of the FPC, the circuit width can be categorized into Top (T) and Bottom (B) circuit lines (Fig. 1). These are the varieties on the horizontal etching.

Currently, the circuit width of the FPC is at higher levels of standard deviation at 0.0017 and 0.0026 millimeters $(\mathrm{mm})$ on the top and bottom circuit widths, respectively. The average values of both circuit widths are at lower levels when compared to the target of $0.10 \mathrm{~mm}$. According to the current process capability study, the sigma level of the FPC manufacturing process is not very satisfactory. Therefore, this etching process should be investigated in order to reach the optimal operating condition. For the preferable responses, this improvement applies four steps of experimental designs and analyses to find out the suitable levels of process variables.

Corresponding Author: Pongchanun Luangpaiboon, Department of Industrial Engineering, Faculty of Engineering, Thammasat University, Pathumthani, 12120, Thailand 
Am. J. Applied Sci., 9 (5): 772-778, 2012

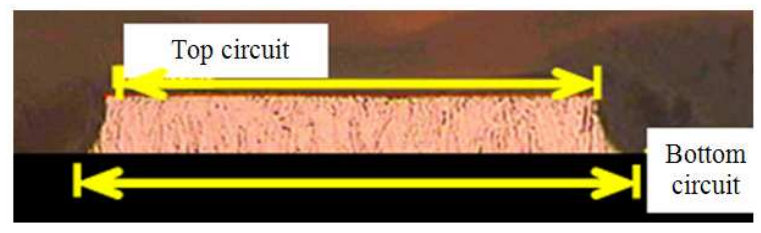

Fig. 1: Crossed section of the circuit width

\section{MATERIALS AND METHODS}

In an optimization, process variables can be both controllable and uncontrollable when applied to industrial experiments. The experiments are run by controlling the current levels of the uncontrollable process variables and then performing all or some of the combinations of the controllable process variable levels in a context of designed experiments with or without replications. Then, a new operating condition of the uncontrollable process variables is chosen and the sequential procedures are repeated. The objective of various industrial experiments is to determine the levels of the designed process variables that optimize a response via these general three steps.

The first is a screening experiment. Its objective is to reduce the many candidate process variables to a relatively few potentially important process variables that influence the process response. This also allows experiments in the following steps to be more efficient and use fewer experimental runs. The second step of steepest ascent (descent) is to bring useful information from the previous step to move through the experimental region in an attempt to get closer to the maximum (minimum). In some cases the process variables and related process limitations form the mathematical programming models such as the Linear Constrained Response Surface Optimization Model (LCRSOM). The last one is an optimization or process mapping. After a success to obtain a small region around the optimum a mathematical model of the system that approximates the true second-order response function is then formed to approximate curvature near the optimum by using statistical analysis of the experimental results. The model can be used to determine optimal operating conditions on the design process variables (Luangpaiboon et al., 2010).

Designed Experiments (DE): Investigation of the Flexible Printed Circuit (FPC) process is relatively hard due to the complexity and number of process variables and multiple responses. Simple designed experiments can take long computational times to explore a complex process and the results mostly depend on the skill and experience of the experimenter. Designed experiments can determine and quantify how the interaction of two or more process variables affects the FPC process responses. It is also easier and faster with the designed experiments to demonstrate their relationships. A completely randomized design is one where the treatments are assigned completely at random so that each experimental unit has the same chance of receiving any one treatment (Brase and Brase, 2011).

Data obtained from the designed experiments can be analyzed using Analysis of Variance (ANOVA). ANOVA collects statistical models which compare means by subdividing the overall observed variance into different combinations. ANOVA shows whether model variance is statistically significant when compared to the experimental variance. Moreover, designed experiments with center design points will detect nonlinearity. Then, if nonlinearity is detected, other tests can be performed to determine which process variables are responsible for the nonlinearity. In most cases, other tests are not needed. A sequential strategy allows the organization to spend money on these extra tests only when they are needed (Ireson and Coombs, 1988).

Steepest Descent Method (SDM): The steepest descent method is one of the simplest and the most fundamental minimization methods for unconstrained optimization. Since it uses the negative gradient as its descent direction, it is also called the gradient method. After a first-order model has been formed, the regression coefficients from this linear model are used to determine the coordinates of related process variables along the path. Through the feasible experimental region the movement of process variables along the steepest descent path is proportional to the magnitude of the regression coefficient, with the direction based on the sign of the coefficient. The path of steepest descent moves a specific distance away from the center of designed process variables in the direction of the minimal response. The minimization procedure of the linear function uses Lagrange multipliers by taking the partial derivatives with respect to each process variable.

Interchangeable Linear Constrained Response Surface Optimization Models (IC-LCRSOM): The response surface methodology or RSM is the combination of mathematical and statistical aspects to optimize the response. It is an empirical modeling technique devoted to the evaluation of relations existing between a group of controlled experimental process variables and the observed results of one or more 
selected criteria (Iqbal and Khan, 2010). Many statistical techniques are used to solve multiple response problems such as overlaying the contours plot for each process response, constrained optimization problems and the desirability approach. In this research, a linear constrained response surface optimization model is recommended due to its simplicity, availability in the software and provides flexibility in giving importance for the specific response. Solving such multiple response optimization problems using this technique involves using a technique for combining multiple responses into interchangeable linear models. The multiple regression model of each response will be set as the objective and remaining regression models will turn to be merely the constraints. The outcomes from all the models will be overlaid and the preferable levels of process variables are then determined.

In this study, the interchangeable linear constrained response surface optimization model (IC-LCRSOM) is proposed to set up a relationship of the linear constrained responses and influential process variables. Originally, linear programs are problems that can be expressed in a canonical form (1).

\section{Minimize ${ }^{\mathrm{T}} \mathrm{M}$ \\ subjecttoAX $\geq \mathrm{B}$ \\ and $X \geq 0$}

where, $\mathrm{X}$ represents the vector of process variables (to be determined), $\mathrm{C}$ and $\mathrm{B}$ are vectors of (known) coefficients and $\mathrm{A}$ is a (known) matrix of coefficients of problem constraints. The expression to be maximized or minimized is called the objective function $\left(\mathrm{C}^{\mathrm{T}} \mathrm{X}\right.$ in this case). The constraints $\mathrm{Ax} \geq \mathrm{B}$ specify a convex polytope over which the objective function is to be optimized (Luangpaiboon, 2011).

\section{RESULTS}

Due to the poor knowledge on process variables influence on multiple quality performance measures, the tests were carried out using a sequential experimental strategy. The studied objective functions or process responses consist of the etched rate $\left(R_{E R}\right)$, the circuit width difference from the target on the top $\left(\mathrm{R}_{\mathrm{TCW}}\right)$ and on the bottom $\left(\mathrm{R}_{\mathrm{BCW}}\right)$. There is no exact customers' specification on the etched rate, but the minimal standard deviation is the most preferable. The target on both circuit widths is at $0.1 \mathrm{~mm}$. This strategy aims to perform a number of experimental design tests that enable a determination of process variables which have significant effects on the process and determination of the optimum. In order to achieve this, the research was conducted by four steps.

The first is to identify the influential process positions that have a significant influence on the objective functions of the top and bottom circuit widths. The next step is to develop designed experiments in order to obtain the new levels of significant process variables affecting the etched rate. Under controlled levels of etched rate, the third is to develop the mathematical model and verification of its adequacy to move toward the optimum via the path of steepest descent based on the circuit width. Finally, it is an analysis of the new operating condition via the effects of different paths of steepest descent on various objective functions in forms of IC-LCRSOM in order to find the optimal working range via the overlaid mapping process.

In the first step, a completely randomized design or one-way Analysis of Variance (ANOVA) was applied to analyze the process responses on circuit widths. The experimental designs were performed to determine the statistically significant process conditions or the capability of measurement system which consist of the pattern and sheet positions. The process positions and feasible ranges are provided in Table 1. In this study, the numerical results shown that on both circuit widths the significant process is the pattern position at the 95\% confidence interval (Table 2). The pattern position is then applied as the designed position in the next step.

In the second step, according to the results from the first step both circuit widths are unbalanced at each level of pattern position, so the second step aims to analyze the etched rate $\left(\mathrm{R}_{\mathrm{ER}}\right)$. A two level experimental design with additional two centre design points was performed to determine the statistically significant process variables of $\mathrm{A}, \mathrm{B}$ and $\mathrm{C}$ (an attribute). The levels of process variables $(\mathrm{A}, \mathrm{B}, \mathrm{C})$ on the centre design points are $(45,3.0,-1)$ and $(45,3.0$, $1)$. Low and high levels including centre design points are selected cover values of feasible ranges in a production line (Table 3). Note that all levels as shown in this research are all coded. At the 95\% confidence interval all sources of variation and their P-Value were shown in Table 4.

According to the results from the analysis of variance, $\mathrm{A}$ is the most important influential process variable, closely followed by B. There was no significant on all interaction effects including the main effect of an attribute process variable of $\mathrm{C}$. From the main effect plot, appropriate levels of process variables $\mathrm{A}$ and $\mathrm{B}$ are set at 60 and 3.1, respectively. When the new operating condition has been applied, there is no improvement on the sample mean of etched responses. 
Am. J. Applied Sci., 9 (5): 772-778, 2012

Table 1: Process positions and their feasible ranges

\begin{tabular}{ll}
\hline Position & Level \\
\hline Pattern & MT, Cen1, Cen2, OP \\
Sheet & S1-S15 \\
\hline
\end{tabular}

Table 2: ANOVA for base line analysis

\begin{tabular}{|c|c|c|}
\hline \multirow[b]{2}{*}{ Position } & \multicolumn{2}{|c|}{ P-Value } \\
\hline & $\mathrm{R}_{\mathrm{TCW}}$ & $\mathrm{R}_{\mathrm{BCW}}$ \\
\hline$\overline{\text { Pattern }}$ & 0.000 & 0.000 \\
\hline Sheet & 0.881 & 0.954 \\
\hline
\end{tabular}

Table 3: Process variables and their feasible and tested levels on the etched rate analysis

\begin{tabular}{llrr}
\hline & & Tested levels & \\
Process & Feasible & -- & High \\
variable & level & Low & 60.0 \\
\hline A & $30-60$ & 30.0 & 3.1 \\
B & $2.0-4.0$ & 2.9 & 1.0 \\
C & Attribute & -1.0 & \\
\hline
\end{tabular}

Table 4: ANOVA with all main and interaction effects

\begin{tabular}{ll}
\hline Sources & P-Value for $\mathrm{R}_{\mathrm{ER}}$ \\
\hline $\mathrm{A}$ & 0.001 \\
$\mathrm{~B}$ & 0.029 \\
$\mathrm{C}$ & 0.371 \\
$\mathrm{~A}^{*} \mathrm{~B}$ & 0.791 \\
$\mathrm{~A}^{*} \mathrm{C}$ & 0.675 \\
$\mathrm{~B}^{*} \mathrm{C}$ & 0.169 \\
$\mathrm{~A}^{*} \mathrm{~B} \mathrm{C}$ & 0.201 \\
Centre point & 0.162 \\
\hline
\end{tabular}

Table 5: Process variables and their feasible and tested levels for circuit width analysis

\begin{tabular}{lllll}
\hline & & \multicolumn{2}{l}{ Tested level } & \\
Process & Feasible & - & \\
variable & level & Low & Center & High \\
\hline B & $2.0-4.0$ & 2.9 & 3.1 & 3.3 \\
D & $3.0-4.0$ & 3.4 & 3.5 & 3.6 \\
\hline
\end{tabular}

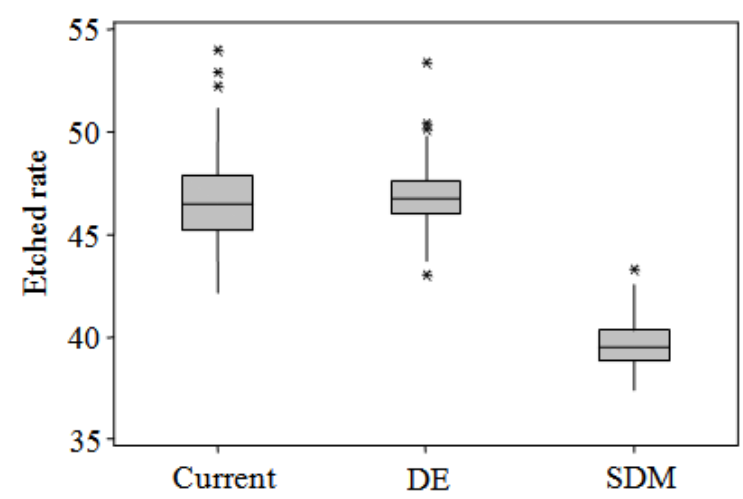

Fig. 2: Box-Whisker Plot of $R_{E R}$

However, its sample standard deviation decreases from 2.033-1.365.
The method of multiple regression analysis at the significance level of 5\% is then applied for statistically significant process variables to determine the most preferable fitted equation of associated process variables of $\mathrm{A}$ and $\mathrm{B}$ on the response of the etched rate. According to the obtained experimental results the developed model is statistically accurate and can be used for further analyses. The fitted first order model in terms of actual process variables is shown Eq. 2:

Expected $\mathrm{R}_{\mathrm{ER}}=63 \cdot 14-0.1708 \mathrm{~A}-13.875 \mathrm{~B}$

From the fitted model, design points are typically carried out one at a time on the path of steepest descent. After a setting in the process variables is determined, there are some steps along the path with the step size of 0.2 before the response of etched rate starts to deteriorate. The preferable levels of process variables $\mathrm{A}$ and $B$ are 60 and 3.8, respectively. When the new operating condition has been applied, the new etched rate is improved with the sample standard deviation of 1.124. In summary, the etched rate has been continually improved with the P-Value of 0.000 (Fig. 2).

In the next step the process variable of $\mathrm{A}$ is then fixed at the suitable level of 60 to maintain the preferred level of etched rate from the previous step. The remaining variable of $\mathrm{B}$, associated with the etching process, returns to be merely a process variable when focused on the responses of both circuit width. Another experiment with B and the additional process variable of $\mathrm{D}$ is then carried out. The low and high levels including centre design points are selected cover values of feasible ranges in a production line to investigate the response or the circuit width difference from the target as shown in Table 5.

Regression coefficients were calculated using the least-square method. Their estimated values including the most preferable fitted equation of associated process variables of $\mathrm{B}$ and $\mathrm{D}$ at the $95 \%$ confidence interval are listed in Table 6 and 7 for all studied objective functions or response on the top $\left(\mathrm{R}_{\mathrm{TCW}}\right)$ and bottom $\left(\mathrm{R}_{\mathrm{BCW}}\right)$, respectively. The relationships of the process variables and the responses in terms of the paths of steepest descent are then determined via the fitted linear multiple regression lines as follow Eq. 3 and 4:

Expected $\mathrm{R}_{\mathrm{TCW}}=0.05392-0.0162 \mathrm{~B}+0.0075 \mathrm{D}$

Expected $\mathrm{R}_{\mathrm{BCW}}=0.1279-0.0342 \mathrm{~B}-0.0035 \mathrm{D}$

The method of steepest descent is then applied to determine the most preferable levels of associated process variables to the responses of $R_{T C W}$ and $R_{B C W}$. 


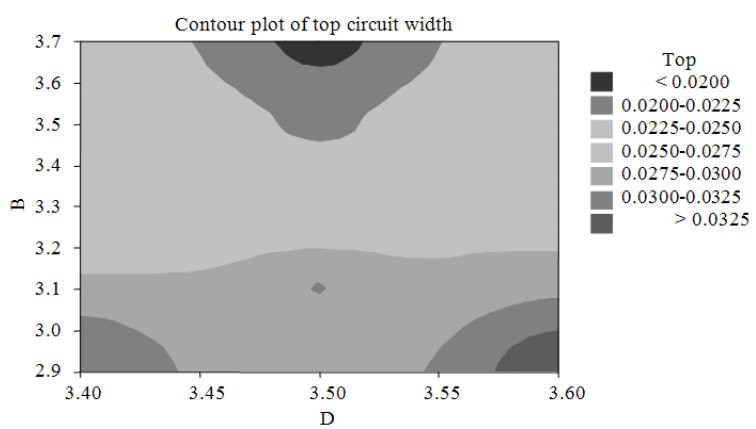

(a)

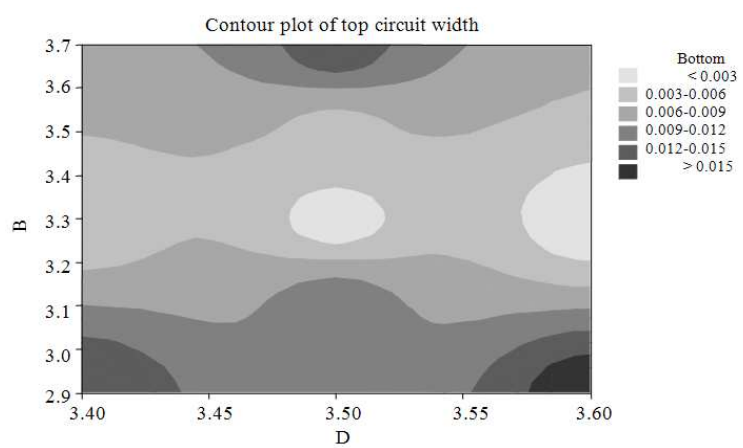

(b)

Fig. 3: Estimated Contour Plots of $\mathrm{R}_{\mathrm{TCW}}$ (a) and $\mathrm{R}_{\mathrm{BCW}}$ (b)

Table 6: Regression analysis including its significant coefficients and ANOVA table on $\mathrm{R}_{\mathrm{TCW}}$

\begin{tabular}{lllcll}
\hline Predictor & Coef & SE Coef & \multicolumn{1}{l}{ T } & P-value & \\
\hline Constant & 0.05392 & 0.007415 & 7.27 & 0.018 & \\
B & -0.0162 & 0.000968 & -16.78 & 0.004 & \\
D & 0.00750 & 0.001936 & 3.87 & 0.061 & \\
Source & DF & SS & MS & F & P-value \\
Regression & 2 & 0.000045 & $22 \times 10^{-6}$ & 148.33 & 0.007 \\
Residual & 2 & 0.0000003 & $15 \times 10^{-7}$ & & \\
Total & 4 & 0.000045 & & & \\
\hline
\end{tabular}

Table 7: Regression analysis including its significant coefficients and ANOVA table on $\mathrm{R}_{\mathrm{BCW}}$

\begin{tabular}{llllll}
\hline Predictor & Coef & SE Coef & T & P-value & \\
\hline Constant & 0.1279 & 0.04287 & 2.98 & 0.096 & \\
B & -0.0342 & 0.005598 & -6.12 & 0.026 & \\
D & -0.0035 & 0.01120 & -0.31 & 0.784 & \\
Source & DF & SS & MS & F & P-value \\
Regression & 2 & 0.000188 & $94 \times 10^{-6}$ & 18.77 & 0.05 \\
Residual & 2 & 0.000010 & $5 \times 10^{-6}$ & & \\
Total & 4 & 0.000198 & & & \\
\hline
\end{tabular}

Table 8: $\mathrm{R}_{\mathrm{TCW}}$ and $\mathrm{R}_{\mathrm{BCW}}$ via the $\mathrm{SDM}$

\begin{tabular}{llll}
\hline $\mathrm{B}$ & $\mathrm{D}$ & $\mathrm{R}_{\mathrm{TCW}}$ & $\mathrm{R}_{\mathrm{BCW}}$ \\
\hline 2.9 & 3.4 & 0.032 & 0.0150 \\
3.3 & 3.4 & 0.026 & 0.0040 \\
2.9 & 3.6 & 0.034 & 0.0170 \\
3.3 & 3.6 & 0.027 & 0.0006 \\
3.1 & 3.5 & 0.030 & 0.0110 \\
3.3 & 3.5 & 0.025 & 0.0010 \\
3.5 & 3.5 & 0.022 & 0.0050 \\
3.7 & 3.5 & 0.019 & 0.0140 \\
\hline
\end{tabular}

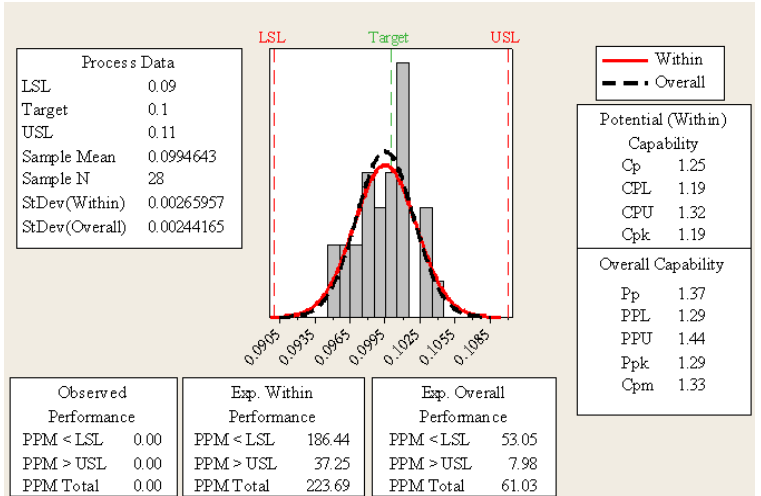

Fig. 4: SDM Performance on $\mathrm{R}_{\mathrm{BCW}}$

The actual step size is determined by the experimenter with a consideration of other practical's or the process knowledge. These experiments will be terminated when there is an increase in responses from the last step. Eventually the experiments arrived to the vicinity of the optimum. From all the design points of current and previous conditions, the estimated relationship of the process variables of $\mathrm{B}$ and $\mathrm{D}$ and the responses, categorized by top and bottom circuit widths, are shown in Fig. 3.

The mathematical programming model is then formulated to minimize the desired response of the circuit width difference from the target. For the top circuit width, the preferable levels of process variables $\mathrm{B}$ and D are 3.7 and 3.5, respectively. For the bottom circuit width, the preferable levels of process variables $\mathrm{B}$ and D are 3.3 and 3.5, respectively (Table 8). Both new operating conditions from the steepest descent are different, but a higher level of the circuit width affects the short circuit defect more seriously on the FPC process. The latter is then used to implement.

After an implementation of the new operating condition (B and D are 3.3 and 3.5, respectively), the sample mean on the top circuit width is improved from $0.074-0.075 \mathrm{~mm}$ and the sample standard deviation from $0.0017-0.0012 \mathrm{~mm}$. The sample mean on the bottom circuit width is improved from 0.097-0.099 mm, the sample standard deviation from 0.0026-0.0024 mm and Cpk increases from 0.85-1.19 (Fig. 4).

In the final step, the experiments aim to analyze both top and bottom circuit widths by using interchangeable Linear Constrained Response Surface Optimization (IC-LCRSOM). All equations (2-4) from previous steps were used to generate the proper levels of process variables on all responses via the overlaid mapping process. The new levels of process variables A, B and D are 60, 3.4 and 3.5, respectively. 
Am. J. Applied Sci., 9 (5): 772-778, 2012

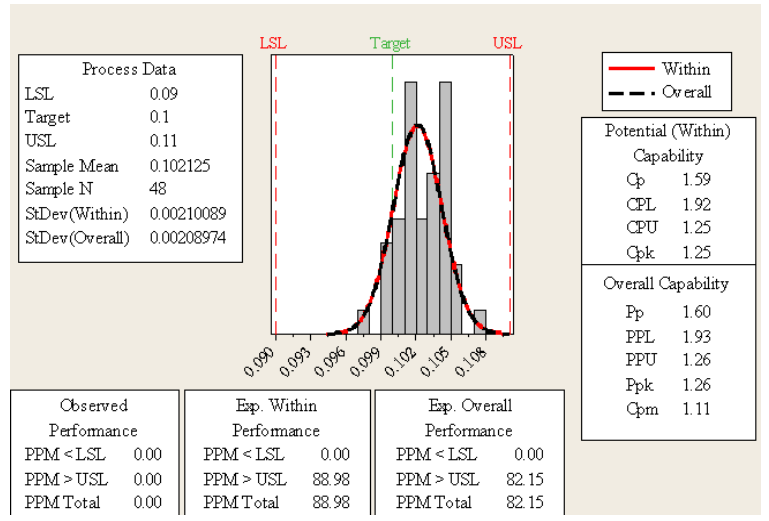

Fig. 5: IC-LCRSOM Performance on $\mathrm{R}_{\mathrm{BCW}}$

When the new operating condition has been applied to the FPC process, the sample mean on the top circuit width is improved from $0.074-0.077 \mathrm{~mm}$ and the sample standard deviation from $0.0017-0.0012 \mathrm{~mm}$. The sample mean on the bottom circuit width is improved from $0.097-0.102 \mathrm{~mm}$ and the sample standard deviation from $0.0026-0.0021 \mathrm{~mm}$. Cpk increases from 0.85-1.25 (Fig. 5).

\section{DISCUSSION}

The current operating condition from the manufacturing process of FPC affects the width of both top circuit and bottom circuit including the etched rate differently. It is quite hard to design the experiments to successfully approach the optimum. Moreover, some design points ended prematurely. Currently the operating condition brings the top circuit width with some distant from target whereas the bottom circuit width close to target. The quality performances of FPC are slightly hard to measure. It should be noted that sequential steps are needed to carry out. This study performed designed experiments to enhance and retain the performance measure of etched rate and continue to improve the circuit width on both top and bottom via the conventional designed experiments, steepest descent and IC-LCRSOM.

During implementations of the steepest descent and IC-LCRSOM the outcomes rely heavily on the assumption of no interaction between influential process variables. The normalized regression coefficient will then be used to move the designs points with some preset step length. This would allow the experimenter to make great moves in the influential process variable toward a region where the optimum lies. When leaping along this path, it is useful to have a rule for deciding when to stop conducting design points along the path of steepest descent. A good rule of thumb is to continue experimenting along the path of steepest descent until some consecutive runs have resulted in an increase in the response.

Often times, due to constraints and multiple responses, it is not feasible to optimize the process via the conventional steepest descent. It would be virtually impossible to successfully run one design point at a time because of the interaction effects among the responses. We have introduced the mathematical model involving some limitations on the process variables to handle the different process responses. After defining how to determine all paths via multiple regression analyses, we propose a framework of overlay mapping for carrying out the solution. Each method has its own advantages and disadvantages. The best method will depend on the particular designed experiments, the starting design points used, the shape of the actual response surfaces, experimenters' experience and results from previous experiments.

\section{CONCLUSION}

The influence of the operating process variables on the FPC manufacturing process has been studied using four experimental steps based on the concepts of designed experiments, steepest descent and ICLCRSOM. Focusing on the last two concepts, empirical models have been developed. They allowed experimenters to determine a correlation between influential process variables and performance indicators or responses, such as etched rate, top and bottom circuit width. The designed experiments are performed and then the steepest descent analysis and interchangeable linear constrained response surface optimization models are applied to investigate the preferable levels of significant process variables in order to improve the quality of both top and bottom circuit widths. Both of experimental models bring the preferable responses of both circuit widths. Though, the experimental results from the SDM seem to be worse. For further research it is very desirable to enhance its procedures by varying step length which enable fast convergence and possess the monotone property.

The mathematical model of IC-LCRSOM, that have been developed, can be used for the selection of operating process variables' proper values in order to obtain the more desired values of key performance indices when compared (Table 9). Especially, the sample mean on the top circuit width is close to the target and there is an increase in Cpk on the bottom circuit width and decrease in the sample Standard Deviation (SD) from customer requirement from all process patterns (Fig. 6). After an actual implementation of the new operating condition from the IC-LCRSOM, the experimental results on top and bottom circuit widths were statistically significant at the $5 \%$ significance level. 


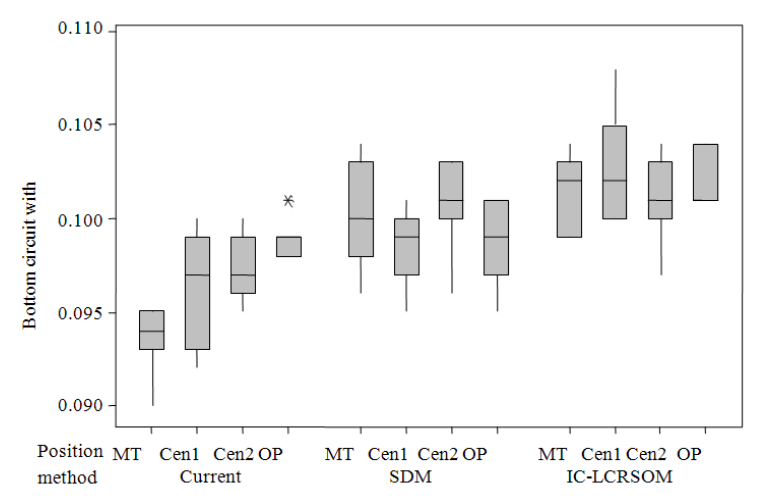

Fig. 6: Box-Whisker plot on $\mathrm{R}_{\mathrm{BCW}}$

Table 9: Key Performance Indices (KPI) on circuit width

\begin{tabular}{lllll}
\hline Circuit width & Condition & Mean & SD & Cpk \\
\hline Top & Current & 0.074 & 0.0017 & -3.03 \\
& SDM & 0.075 & 0.0012 & - \\
\multirow{3}{*}{ Bottom } & IC-LCRSOM & 0.077 & 0.0012 & - \\
& Current & 0.097 & 0.0026 & 0.85 \\
& SDM & 0.099 & 0.0024 & 1.19 \\
& IC-LCRSOM & 0.102 & 0.0021 & 1.25 \\
\hline
\end{tabular}

Observe that only first order information on the responses has been used. Therefore, an interesting topic for future research is an elaboration of a higher order mathematical model for a multiple response optimization. It is also suggested that the company extend this systematic approach to different machine and combinations, so that the optimal settings can be applied to all machine and combinations.

\section{ACKNOWLEDGMENT}

This work was supported by the Higher Education Research Promotion and National Research University Project of Thailand, Office of the Higher Education Commission.

\section{REFERENCES}

Brase, C.H. and C.P. Brase, 2011. Understandable Statistics: Concepts and Methods. 10th Edn., Cengage Learning, Boston, MA., ISBN: 0840048386, pp: 832.

Coombs, C.F., 2007. Printed Circuits Handbook. 6th Edn., McGraw-Hill, New York, ISBN: 0071467343, pp: 1000.

Iqbal, A.K.M.S. and A.A. Khan, 2010. Modeling and analysis of MRR, EWR and surface roughness in EDM milling through response surface methodology. Am. J. Eng. Applied Sci., 3: 611619. DOI: 10.3844/ajeassp.611.619.

Ireson, W.G. and C.F. Coombs, 1988. Handbook of Reliability Engineering and Management. 2nd Edn., McGraw-Hill, New York ISBN: 007032039X, pp: 608.

Luangpaiboon, P., 2011. Constrained response surface optimization for a laser beam welding process. J. Math. Stat., 7: 5-11. DOI: 10.3844/jmssp.5.11

Luangpaiboon, P., Y. Suwankham and S. Homrossukon, 2010. Constrained response surface optimization and taguchi methods for precisely atomizing spraying process. IAENG Trans. Eng. Technol., 5: 286-300. DOI: 10.1063/1.3510555

Tong, J.P., C.F. Tsung and B.P.C. Yen, 2004. DMAIC approach to printed circuit board quality improvement. Int J. Adv. Manuf. Technol., 23: 523-531. DOI: 10.1007/s00170-003-1721-z 